\title{
Where do we stand with asthma phenotypes derived from data- driven methods? A systematic review
}

\author{
Rita Amaral, Tiago Jacinto, Bernardo Sousa-Pinto, João Fonseca
}

European Respiratory Journal 2019 54: PA1124; DOI: 10.1183/13993003.congress-2019.PA1124

\begin{abstract}
Asthma phenotypes can be refined using methods without a priori assumptions (data-driven). We aimed to describe asthma phenotypes derived with data-driven methods, using variables easily measurable in a clinical setting, and to summarize their consistency.
\end{abstract}

Systematic review of the literature using PubMed, Scopus and Web of Science, with no date/language restrictions. We included studies reporting adult asthma phenotypes applying datadriven methods with variables that can be easily collected in a clinical setting. Exclusion criteria were studies focusing exclusively on children and/or genotyping and theory-driven methods. Studies were assessed by two independent reviewers.

Of the 3,757 articles identified, 52 were included. The most frequent number of phenotypes identified per study was 4 and 5 phenotypes. Most of the studies focused on patients from specialized centers $(n=41 ; 79 \%)$. Variables used for phenotyping substantially differ according to the samples' characteristics, data availability, study aim and data sources. Variables were matched and categorized into six groups: clinical( $n=38)$, functional( $n=32)$, socio-demographic $(n=25)$, inflammation $(n=23)$, atopy $(n=19)$, and other $(n=9)$. Studies evaluating the consistency of phenotypes(n=15;29\%) followed $\geq 1$ of the criteria: longitudinal stability, cluster repeatability, reproducibility, and validity.

Data-driven methods have been increasingly used to derive asthma phenotypes, particularly in specialized centers. However, there's significant heterogeneity that requires clinical expertise and 
statistical know-how for their evaluation. Studies with population-based samples and reporting longitudinal consistency of data-driven phenotypes are scarce. 